Original article

\title{
Fasting duration in patients undergoing elective surgery
}

\author{
NajmehValizadeh Zare ${ }^{1}$, Maryam ShaghaeeFallah ${ }^{2, *}$
}

(Received: 22 Sep 2013; Accepted: 2 March 2014)

\begin{abstract}
Background and Purpose: Preoperative fast is the essential part of preoperative care for those patients who are candidates for elective surgery. Fast prolongation results in side effects such as: sweating, irritability, decrease in blood sugar and restlessness. Despite the noticeable changes in fasting guidelines and their application in many countries, it seems that in Iran, these rules are not applicable very much. This research aim to detect the fasting time in patients as the candidates for elective surgery.

Methods: This descriptive study was conducted on 124 patients as the candidates for elective surgery in teaching hospitals in Mashhad. Data have been gathered by a researchers-made questionnaire after confirming its validity and reliability by being presented in the operation rooms. The data were analyzed by descriptive statistics.

Results: The research findings indicate that 53.6 percent of the patients were female. The patients ranged 2-69 years old. Minimum fasting time was 3 hours and the maximum $96 \mathrm{~h}$ with the average of $12 \mathrm{~h}$. The maximum waiting time after being transferred to the operating rooms till the operation starting time was $4 \mathrm{~h}$.

Conclusion: The results denote the patients of different age groups endure long fasting period considerably affecting the illness process and surgery outcome. Some of the reasons could be attributed to the staff's lack of knowledge about the guidelines, not having the scheduled transfer for the operation rooms and the patients being afraid of anesthesia side effects like vomiting and nausea.
\end{abstract}

Keywords: Fasting, Surgery, Waiting time

\section{Introduction}

Pre- surgery fasting is considered as a golden standard to moderate stomach acidity so that it decreases the mortality and morbidity of pulmonary aspiration used for many years in all age groups $(1,2,3)$.

Despite recommendations given to the contrary, the patients are still directed to fast for six to eight $\mathrm{h}$ even longer before the planned surgery. This is not beneficial for the patients, but fits the operating room systems (4).
In the era of evidence-based medicine, however, there are no scientific reasons to keep a patient in prolonged preoperative fasting. This routine has been questioned and proved to be unnecessary for most patients. It seems that the overnight fasting routine has to come to an end in most modern medical societies, at least regarding the recommendation of clear fluids with carbohydrates. However, the dogma of NPO after midnight may last for more years or decades to come despite

${ }^{1}$ Jundishapur Nursing\& Midwifery School, Ahvaz University of Medical Sciences, Ahvaz, Iran

${ }^{2, *}$ Corresponding author: Nursing \& Midwifery School, Mashhad University of Medical Sciences, Mashhad, Iran. Email: shaghaeem@ mums.ac.ir 
the overwhelming evidence of safety and benefits in reducing preoperative fasting time. Seymour (2000) stated that the tradition and custom often dictate preoperative fasting regimens rather than the individual patients' needs (5).

Smith (1997) found out that the patients fasting for longer than the optimum period can be detrimental to their health. However, Green et al (1996) \& Williams (1999) confirmed that this is a common practice. Furthermore, Green et al (1996) \& Hung (1992) suggested that excessive fasting will not produce the optimum gastric environment but may cause dehydration, electrolyte imbalance, malnutrition, nausea, hypothermia, fatigue and irritability. Splinter and Schreiner (1999) asserted that due to their reduced body size, children can become dehydrated and as a result develop an electrolyte imbalance, feel nauseous and experience irritability and fatigue very quickly.Hausel et al (2001) discovered that alleviating thirst, hunger and anxiety before elective surgery improved the patients' comfort and postoperative recovery (4).The findings of Seymour (2000), Pandit \& Pandit (1997) suggested that it is not happening. If nurses are not up-to-date with the current research and practice, it is not a legal defense against misconduct. If patients have fasted for too long or not sufficiently and suffer discomfort or problems during the anesthetic procedure as a result, this can become a legal matter (4).

Statistics show that death due to aspiration is one the rare harms of modern anesthesia. The high prevalence of aspiration reported by Mendelson in 1946 was 0.15 percent that is five times more than the one ( 0.04 percent) reported by Olsson et al,.Tirt et al, $(0.01 \%)$ and Warner et al 's $(0.04 \%)$ finding. Digestion period is subject to the kind of consumed food, the fasting time around 6 $\mathrm{h}$ is enough for making sure of gastric depletion, and it has been proved that such a long stretching of fast is unnecessary for liquids consumption before surgery $(6,7)$. Liquids and water remain in stomach for only 12 minutes and surveys have revealed that the volume and PH of stomach or plasma osmolarity would not be affected by unlimited water intake until $2 \mathrm{~h}$ prior to surgery (8).

Long time fast affects the patients' physical and mental health and leads to many harms and restlessness before and after the surgery (1). Recently the studies have shown that pre- surgery overnight fasting will have meaningful negative effects on surgery recovery. These statistics have proved an increase in resistance to insulin and the decrease of muscle mass after surgery as one of the consequences of long starvation before elective surgeries (2).

The major effects of long fasting can be divided into physical and mental signs. The physical signs include dehydration, headache, hypovolamia, hypoglycemia, electrolyte disorders, nausea, vomiting and malnutrition. Mental sings cover distress, unpleasant experience, irritability, agitation and confusion. These evidences appear more quickly among the younger and the elderly patients and are more harmful. To prevent these indications could avoid surgery cancelation and anesthesia $(8,1)$.

According to American Society of Anesthesia (ASA) guidelines codified in 1999, patients who do not have any special disease or are not pregnant should not have solid food for $6 \mathrm{~h}$ and liquids for $2 \mathrm{~h}(1)$. This instruction is based on the studies suggesting that respiratory aspiration in modern anesthesia is not common, and there is a little relationship between preoperative fasting and stomach $\mathrm{PH}$ and volume, and clear liquid consumption 2-4 h before surgery might lower stomach volume $(10,11)$.

Strict fasting rules implementation will lead to usual routine fasts after midnight (6). Moreover, experiences manifested that the fasting period is usually too long and its starting point is different from evening (after having dinner) to midnight, and it seems that this period is not related to surgery time and the patient's condition. Almost all patients having elective surgery obey the rule of "midnight fasting". Hence the following study was done in order to determine the fasting time for those patients undergoing elective surgery in Mashhad. 


\section{Materials and Methods}

This descriptive research was done in 2011 with the aim to determine fasting time in three hospitals in Mashhad. Samples were all patients on elective surgery list. Including criteria were being in the elective surgery schedule and candidate for general anesthesia. Sampling was done in general operating rooms every day for 2 months from 7 am to $2 \mathrm{pm}$, except Thursdays and Fridays as being specific to washing and emergency surgeries.

124 patients from these three centers were included in this cross-sectional study. The researcher's specific fasting duration and probably the side effects due to fasting, then the correct time of being transferred to the operating room, anesthesia induction and the end of it have documented by the researcher. In addition, all of the information collected by one sample was excluded because of simultaneity. Sampling went on for two months. It is worth mentioning that the information was collected from the patients ten minutes before the start of the surgery and there was only one onsite interviewer.

Sample size calculated 124 by using this formula $n=\frac{Z_{1-\alpha / 2}{ }^{2} S^{2}}{d^{2}}$ was determined by the guide's studies. No standard questionnaire was obtained, information was obtained by the researcher- made questionnaire based on different studies about fasting, and then content validity was confirmed by the anesthesia experts. The pilot study was done with 20 samples. Tool reliability was confirmed by the use of Alpha-Cronbach reliability calculated as 0.85 . The questionnaire consisted of two parts: demographic data and fasting time data completed by the researcher after attending the operating rooms and interviewing the patients before the surgery or after cancelling it.

Findings were analyzed by SPSS software version 11.5 and the descriptive statistic including mean, maximum and minimum, relative frequency and Kruskal Wallis test were used in order to assess the significant relationship between age and fasting period.

\section{Results}

The age mean was $36.5 \pm 17.5$, the maximum age range $(30 \%)$ was between 15-25. 47 percent were female, 88 percent had no medical history, 4 percent were diabetic and 3.2 had hypertension. The highest surgeries $(40 \%)$ were related to the orthopedic surgeries. 55 percent of the patients were transferred to the operating room between 7 to 8 in the morning while 9.7 percent of them underwent surgery immediately after transfer. The mean of waiting time from transfer to the operating room to start the surgery was $1.1 \pm 1 \mathrm{~h}$. Only 15 percent of the patients were aware of their surgery time and for the rest of the cases, the following morning, it was announced to the patient by the nursing staff.18.5 percent were fasting less than $8 \mathrm{~h}, 16$ percent between $11-12 \mathrm{~h}$ and the rest were fasting more than $12 \mathrm{~h}$. They abstained from solid food and liquids.

Table1. Frequency of different surgery

\begin{tabular}{lcc}
\hline Kind of surgery & Frequency & $\mathbf{\%}$ \\
\hline Gynecology & 17 & 13.7 \\
Orthopedic & 50 & 40.3 \\
Abdomen & 11 & 8.9 \\
Eye & 11 & 8.9 \\
Urology & 11 & 8.9 \\
Plastic & 3 & 2.4 \\
E,N,T & 12 & 9.7 \\
Lumbar disk & 4 & 3.2 \\
Other & 5 & 4.0 \\
Total & 124 & 100 \\
\hline
\end{tabular}

Generally speaking, fasting time varied from 3 to $96 \mathrm{~h}$. Most of the patients (38.2 percent) started their fasting from 11 to 12 p.m. and 18.7 percent started it from 9 to 10 p.m. Only one patient was fasting for $96 \mathrm{~h}$ because of having several surgeries cancelation. Fatigue and headache were the most common complications faced by $33 \%$ of the patients. Others had no difficulty afterwards.

11 surgeries were canceled after transferring the patients to the operating room because of some changes in the patients' health status, the equipment not being 
prepared or last surgery being protracted. In addition, all patients fasted between 9-12 h. Kruskal Wallis test did not show any significant differences between the fasting time, age $(p$ value $=0.714)$, and the kind of surgery $(\mathrm{p}$ value $=0.79)$.

\section{Discussion}

Regardless of the evidence based research cases and the Association Society of Anesthesia (ASA) guidelines (12), the average of fasting time in this study was 12.7 h. This finding is similar to Eduardo's results. He found that, however, that the prescribed $6-8 \mathrm{~h}$ of fasting is usually prolonged to $12-16 \mathrm{~h}$ for various reasons (5). Prolonged fasting triggers a metabolic response that facilitates gluconeogenesis and increases the organic response to trauma. Katherine (2003) pointed out that if emphasis is made on fasting time, fortunately this period will be longer that standard suggested. She believes the hospital policy and delay the surgery is responsible for this evident. If nursing and medical staff assist with the ward staff in setting the order of the operating lists, the unnecessary preoperative fasting will not happen (5). Krenshow \& Winslow (2008) (6), Bariel \& Portman (2007) (15) and Watchel (2009) (20) reported fasting time more than $10 \mathrm{~h}$.

In this research, some of the reasons for such long fasting time might be the fact that apart from obeying the rule of fasting time by the patients, there is no exact schedule for operation time. In addition, since patients are afraid of vomiting and nausea and have no idea of the exact time of being transferred to the operating room, they would abstain from food consumption even drinking. These consequences originate from the surgery department manager's not paying attention to the necessities of the scheduled surgery, and the training staff not educating the patients.

Statistics show the observing the standard fasting time according to ASA guidelines facilitates postsurgery process and reduces hunger, thirst, anxiety, and vulnerability (13). Breuer et al. (2010) stated that fasting after midnight is traditionally recommended by 7 percent of German anesthesiologists (14).
According to the research, the reason for applying new fast guidelines is to feel comfortable after surgery and increase the patient's satisfaction. On the contrary, there are some opposite ideas supporting the risk of aspiration and low flexibility of the operating room management (14). Furthermore, others have indicated that the patient's average fasting time was $14 \mathrm{~h}$ in the case of using solid foods, $12 \mathrm{~h}$ in the case of liquids, and some of them fasted more than $37 \mathrm{~h}$. These findings are applicable to the current study $(15,1)$. In 2006, the study finding of Watchel \& Dexter on 382 patients' fasting time showed that the average fasting time was $12 \mathrm{~h}$ for solids and $7 \mathrm{~h}$ for liquids (20). Moreover, a study was conducted on 60 hysterectomy volunteer patients in Tabriz-based Medical University, that the average fasting time for the patients in this study was 12-16 h (16).

Several clinical trials and meta-analyses on healthy individuals on the elective surgery lists proved that the consumption of water and clear liquids, especially carbohydrates $2 \mathrm{~h}$ before surgery not only did not enhance the volume and acidity of stomach secretion, but also decreased resistance to insulin and surgery stress and made the patients feel at ease $(12,7)$.

Feria et al., in their study divided adult women chosen for elective surgery into two groups. The first group fasted for $8 \mathrm{~h}$ before surgery and the second one drank $200 \mathrm{ml}$ liquid containing 12.5 percent maltodextrine $2 \mathrm{~h}$ before surgery. Results denoted that short fasting period lessens insulin resistance and organic response to trauma (5).

In 2009, Ghareshi et al., did a clinical trial on 411 pregnant women selected for elective cesarean. The case group was taking $150 \mathrm{ml}$ of carbohydrate liquid orally 1 hour before surgery while the control group took nothing. Results showed that only one case in both groups had regurgitation and there was not any incidence of regurgitation developments in general anesthesia lung aspiration. The findings by Malty et al., manifested that there was no difference between stomach volume and $\mathrm{PH}$ in overweight patients who took $300 \mathrm{ml}$ fluids orally $2 \mathrm{~h}$ before surgery and the other ones fasting since 
midnight (14).

In the current study, there was no appreciable distinction between the fasting times in different age groups, which means fasting time in all age groups are similar, whereas based on different studies, long fasting period especially in children and old adults could affect the recovery trend and comfort feeling after surgery. Yurtcu et al., (2009) conducted a research on 80 children ranging 1 to 10 years old and found out those children who had liquids $2 \mathrm{~h}$ before the surgery could tolerate surgery stress better than the other ones (18).

Also a meta-analysis done by Klemetti et al., (2010) \& Brady et al., (2009) demonstrated that the children who were allowed to drink fluids till $2 \mathrm{~h}$ before the surgery tolerated hunger and thirst better than those fasting till midnight and were calmer. There was no difference between stomach $\mathrm{PH}$ and volume in the two groups (19).

\section{Conclusion}

Despite the negative effects of long fasting, research results show that the statement of "NPO after midnight" is still widely used regardless of the patient's character, surgery techniques and surgery time.

Therefore with respect to the current study and the other studies in this field, it seems that due to the fasting time observation especially in high risk groups, having short fasting time could prevent the related harms. The following recommendations are based on the recent survey and literature review:

- Using cards with different colors to determine kind of fasting (solid and fluid);

- Training staff and having educational posters related to the latest guidelines and research about fasting in surgery wards;

- Educating patients about the surgery time \& type. Because a surgery causes the health status stress, patients are more sensitive to their health condition; so educating patients could be effective to correct fasting implementation before surgery. For example, in this research, some patients fasted too long and refused consuming, especially fluids because of being afraid of anesthesia harms and surgery cancellation;

- Emphasizing on the importance of long fasting;

- Interaction between surgery nurses, surgeons and anesthesiologists in relation to the newest fasting guidelines;

- Considering age as a risk factor for prolong fasting and patients with contextual disease;

- Coordinating with surgeons to set a schedule for transferring patients to the operating room and starting fasting time according to the prepared list.

\section{Conflict of Interests}

The authors declare that they have no competing interests.

\section{Author's Contributions}

M. Shaghaee Fallah and N. Valizadeh Zare have contributed to designing, writing, revising and approving the final manuscript. Data collection and statistical analysis have been performed by the authors.

\section{Acknowledgment}

We appreciate of the patient and our student Miss Reyhani that helped us to collected data and the staff of these three educational hospitals in Mashhad.

\section{References}

1. Anderson M, Comrie R. Adopting Preoperative Fasting Guidelines. AORN Journal 2009; 90(1): 73-80.

2. Portic A, Turina D, Matanic D,Spanjol J, ZuvicButorac M, Sustic A. Preoperative feeding Effect on gastric emptying following spinal anesthesia: a randomized controlled trial. Wien Klin Wochenschr. Springer-Verlag 2010; 122(1-2) :50-53.

3. Brady M, Kinn S, Ness V, O'Rourke K, Randhawa N, Stuart P. Preoperative fasting for preventing preoperative complications in children. Cochrane Database Syst Rev 2009; 7(4): 1-136.

4. Webb K. What are the merits \& demerits of preoperative 
fasting? Nurs Times 2003; 99(50):32-33.

5. Eduardo J, Nascimento A, Borges D. Reducing preoperative fasting time: A trend on evidence. WJGS 2010; 2(3): 57-60.

6. Crenshaw J, Winslow E. Preoperative Fasting Duration and Medication Instruction: Are we improving? AORN Journal 2008; 88(6): 963-973.

7. Marty K, Vater Y, Hunter C, Ross B. Preoperative fasting after soft drink intake: 2 hours may be enough. J Anesth 2002; 16(2) :179-180.

8. Subrahmanyam M, Venugopal M. Perioperative fasting: A time to relook. Indian J Anesth 2010; 54(5): 374-375.

9. Graterl J, Clayton T. Pulmonary aspiration. Anaesth Intensive Care 2010; 11(10): 447-448.

10. Lorch A. Implemention of fasting guidelines through nursing leadership. Nurs Times 2007; 103(18): 30-31.

11. American Society of Anesthesiologist. Practice Guidelines for Preoperative Fasting and the Use of Pharmacologic Agents to Reduce the Risk of Pulmonary Aspiration: Application to Healthy Patients Undergoing Elective Procedure. Anesthesiology 2011; 114(3): 495-511.

12. Maltby J, Pytka S, Watson C, McTaggart R ,Fick G. Drinking $300 \mathrm{ml}$ of clear fluid two hours before surgery has no effect on gastric fluid volume and $\mathrm{pH}$ in fasting and non-fasting obese patients. Can J Anesth 2004; 51(2): 111-115.

13. Ghorayshi Z, Ashori V.R, Aminzadeh F, Mokhtari M. The Clinical Trial on Oral Fluid Intake Effects an Hour before Cesarean on Regurgitation Incidence. Daneshvar medicine. Journal of Shahed University 2009; 16(83):1-6.

14. Breuer JP, Bosse G, Seifert S, Prochnow L, Martin J, Schleppers A, et al. Preoperative fasting: a nationwide survey of German anesthesia departments. Acta Anaesthesiol Scand 2010; 54(3): 313-20.

15. Baril P, Portman H. Preoperative fasting: knowledge and perceptions. AORN Journal 2007; 86(4): 609-612.

16. Afhami M.R., Hasanzadeh Salmasi P. Effect of Preoperative Fasting on Hemodynamic Status of Elective Hysterectomy Patients with General Anesthesia. Journal of ArmaghaneDanesh 2007; 12(4): 17-23.

17. Klanarong S, Suksompong S, Hintong T, Chau-In W, Jantorn P, Werawatganon T. Perioperative Pulmonary Aspiration: An Analysis of 28 Reports from Thai Anesthesia Incident Monitoring Study (Thai AIMS).J Med Assoc Thai 2011; 94(4): 457- 64.

18. Yurtcu M, Gunel E, Sahin TK, Sivrikaya A. Effect of fasting and preoperative feeding in children. WJG 2009; 15(39): 4919-4922.

19. Klemetti S, Kinnunen I, Suominen T, Antila H, Vahlberg T, Grenman R, et al. The effect of preoperative fasting on postoperative thirst, hunger and oral intake in pediatric ambulatory tonsillectomy. JCN 2010; 19(34): 341-350.

20. Wachtel R, Dexter F. Reducing surgical patient fasting times. AORN 2009; 89(5):830-831. 